\title{
In vivo confocal Raman spectroscopy for intrinsic aging and photoaging assessment
}

\author{
Livia de Vasconcelos Nasser Caetano ${ }^{\mathrm{a}, *}$, Thiago de Oliveira Mendes ${ }^{\mathrm{b}}$, Edileia Bagatin ${ }^{\mathrm{a}}$, \\ Helio Amante Miot ${ }^{c}$, Juliana Laudiceia Marques Soares ${ }^{a}$, \\ Milvia Maria Simoes e Silva Enokihara ${ }^{a}$, Airton Abrahao Martin ${ }^{\mathrm{d}}$ \\ a Universidade Federal de São Paulo - UNIFESP, Department of Dermatology, Rua Borges Lagoa 508, Zip Code: 04038-001, Vila Clementino, São Paulo, SP, \\ Brazil \\ ${ }^{\mathbf{b}}$ Universidade do Vale do Paraiba - UNIVAP, Institute of Research and Development, Laboratory of Biomedical Vibrational Spectroscopy, LEVB, Avenida \\ Shishima Hifumi, 2911, Zip Code:12244-000, São José dos Campos, SP, Brazil \\ " Universidade Estadual Paulista "Julio de Mesquita Filho" - UNESP, Department of Dermatology and Radiotherapy, Campus Universitário de Rubião Jr., Zip \\ Code: 18618-000, Botucatu, SP, Brazil \\ d Universidade Federal do Piauí, Campus Universitário Ministro Petrônio Portella, Zip Code: 64049-550, Bairro Ininga. Teresina, PI, Brazil
}

\section{A R T I C L E I N F O}

\section{Article history:}

Received 5 December 2016

Received in revised form 9 June 2017

Accepted 14 July 2017

\section{Keywords:}

High frequency ultrasound

Histology

Photoaging

Raman spectroscopy

Skin aging

\begin{abstract}
A B S T R A C T
Background: In vivo confocal Raman spectroscopy is a non-invasive method to assess either the epidermis or the dermis composition. Few studies have focused on dermis collagen alterations through intrinsic aging and photoaging.

Objective: This study evaluated the in vivo Raman spectra from the dermis of a photoexposed site versus a non-photoexposed region in different age groups, and evaluated the correlation between peak intensities and age, photoaging score and the amount of collagen assessed with histology and high frequency ultrasound (HFUS).

Methods: Fifteen volunteers aged 28-82 years were divided into three groups according to forearm photoaging degree. In vivo Raman spectra from the dermis were collected on the dorsal forearm (chronically photoexposed skin) and on the proximal medial arm (non-photoexposed skin). Crosssectional images of the skin were obtained using a $20 \mathrm{MHz}$ ultrasound unit exactly on the same sites, which were further submitted to punch biopsies for histologic study (collagen I immunohistochemistry, picrosirius red staining and Verhoeff). Principal Component Analysis (PCA) and Orthogonal Partial Least Squares Discriminant Analysis (OPLS-DA) were taken in the spectral region of $796 \mathrm{~cm}^{-1}-996 \mathrm{~cm}^{-1}$ to determine its potential to discriminate between different groups. The Spearman rank correlation coefficient of individual peak intensities and ratios with age, clinical score and the amount of collagen assessed by ultrasound and histology were calculated.

Results: PCA of pairs of groups and OPLS-DA could discriminate the intrinsically from the photoaged skin and the young group from the elderly one, with important contribution of the $938 \mathrm{~cm}^{-1}$ and $855 \mathrm{~cm}^{-1}$ peaks intensities. The intensity of the peaks in $855 \mathrm{~cm}^{-1}$ and/or $938 \mathrm{~cm}^{-1}$ presented moderate correlation with age $(\mathrm{rho}=0.579, p=0.049)$ and moderate to high inverse correlation with HFUS echogenicity (rho $=-0.710, p=0.010)$ and collagen I immunohistochemistry (rho $=-0.833, p=0.005)$ in the nonphotoexposed region. The $\mathrm{I}_{1275} / \mathrm{I}_{1450}$ intensities ratio presented moderate to high correlation coefficients with age $(\mathrm{rho}=-0.730, \mathrm{p}=0.007)$, photoaging score $(\mathrm{rho}=-0.594, \mathrm{p}=0.042)$, HFUS echogenicity (rho $=0.760, \mathrm{p}<0.001$ ) and histology (collagen I immunohistochemistry (rho=0.643, p=0.024), picrosirius ( $(\mathrm{pho}=0.773, \mathrm{p}=0.005)$ and Verhoeff $(\mathrm{rho}=-0.727, \mathrm{p}=0.011)$ ) in the photoexposed site. Conclusion: The wavenumber region between 798 and $994 \mathrm{~cm}^{-1}$ is useful for the analysis of dermal collagen alterations through the intrinsic aging process, while photoaging is better assessed by the $\mathrm{I}_{1275} /$
\end{abstract}

\footnotetext{
* Corresponding author.

E-mail addresses: caetano.livia@gmail.com (L. de Vasconcelos Nasser Caetano), eadthiago@gmail.com (T. de Oliveira Mendes), edileia_bagatin@yahoo.com.br (E. Bagatin), heliomiot@gmail.com (H. Amante Miot), julianalmsoares@hotmail.com (J.L. Marques Soares), milvia.enokihara@yahoo.com.br (M.M. Simoes e Silva Enokihara), airton.a.martin@gmail.com (A. Abrahao Martin).
} 
$\mathrm{I}_{1450}$ intensities ratio. This is the first skin aging study to show a correlation between Raman peaks and the amount of collagen assessed by HFUS and histology.

(c) 2017 Japanese Society for Investigative Dermatology. Published by Elsevier Ireland Ltd. All rights reserved.

\section{Introduction}

Vibrational spectroscopic methods are employed in medical research to analyse single cells to whole tissues, with high sensitivity to biochemical composition and the advantage of being a non-destructive, non-invasive and label-free techniques [1].

When light interacts with matter, part of it is deflected from the direction of the incident electromagnetic wave (light scattering). The dominant type of light scattering is Rayleigh or elastic scattering, which involves no energy transfer: the scattered light has the same frequency as the incident light. Raman spectroscopy relies on the inelastic interaction between light and matter: a minor part of the deflected light has a frequency different from the incident light, because energy is transferred during the scattering event. The resultant spectra are characterised by shifts in wave numbers (inverse of wavelength in $\mathrm{cm}^{-1}$ ) from the incident frequency - Raman Shift. Spectral bands are molecule-specific and provide direct information about the biochemical composition. These bands are sensitive to molecular structure, conformation, and environment [1,2].

Raman spectroscopy has been used for skin research both in vivo and ex vivo. The methods employed in the first in vivo researches were not able to separate the signal contributions from the different skin layers [3]. Caspers et al. described a confocal Raman spectroscopy system designed for the measurement of the different layers of the skin in vivo, obtaining characteristic spectra from the stratum corneum, from the viable epidermis and from the dermis separately [4].

Most in vivo studies have focused on epidermis assessment, either for composition study [5] or for permeation evaluation [6]. The confocal Raman spectrometer requires short laser exposure time (Integration time) for epidermis analysis. The deeper layers of the skin, such as the dermis, need much longer integration time to obtain high quality signal-to-noise ratio spectra, because the deeper the measurement depth, the weaker the Raman scattering is. However, with proper adjustment of the laser exposure time, laser potency and number of accumulations, the signal-to-noise ratio is improved and the dermis layer is studied in vivo, apart from the epidermis structures [7].

From the Raman spectroscopy studies that explore the dermis, few have focused on skin aging [7-11]. Gniadecka et al. [8] used an ex vivo FT-Raman spectroscopy system to study intrinsic aging and photoaging. The young photoexposed and non-photoexposed skin presented similar protein structures and water contents, predominantly bound to other macromolecules. The chronologically-aged region presented a similar water structure to young skin, with minor changes in the protein structure. The photoaged skin presented a downshift of both of the Amide I and Amide III bands, and a decrease in amide III intensity, which could reflect an increased degree of collagen packing. Besides, the non-macromolecule-bound water was increased in this region. Nakagawa [7] obtained similar results regarding water content in elderly photoexposed skin using an in vivo confocal Raman Spectroscopy system.

Nguyen [10] and Téllez [11] evaluated only the non-photoexposed skin, ex vivo and in vivo, respectively. The first group confirmed that the $938 \mathrm{~cm}^{-1}$ band rises with increasing environmental relative humidity and decreases with increasing temperature (which leads to evacuation of free and bounded water), but with no difference between the two age groups [10]. The second research found lower integrated intensity ratio values between the $938 \mathrm{~cm}^{-1}$ and $922 \mathrm{~cm}^{-1}$ bands in the young group compared to the elderly group, and even lower results for a diabetic elderly group [11].

Gonza'lez et al. [9] first attempted to compare Raman spectroscopy findings and skin photoaging histology. The percentage of elastic fibres in photoaged skin correlated with the second component (PC2) of Principal Component Analysis (PCA) of the in vivo Raman spectra obtained from the same sites. However, they do not discuss which spectral regions or which peaks are more prone to contribute to this association.

The objective of this study was to compare the in vivo Raman spectra of the dermis in a photoexposed versus a non-photoexposed region in different age groups. The correlation between selected peak intensities and the amount of collagen assessed by histology and high frequency ultrasound was also tested.

\section{Material and methods}

A cross-sectional study was performed including patients from the department of dermatology of the Federal University of São Paulo (UNIFESP), Brazil. This study protocol was approved by the Ethics Committee under the CAAE number: 10309112.4.0000.5505. Consent form signatures were obtained. Standardised photographs of the right forearm of each subject were randomised for scoring by one dermatologist according to the clinical scale for photoaging evaluation of forearm skin, as described elsewhere [12]. Subjects were divided according to their age and forearm skin photoaging degree: Group 1 or light photoaging Group (score from 0 to 30 and age between 25 and 50 years), Group 2 or moderate photoaging Group (score from 31 to 70 and age between 50 and 60 years), and Group 3 or advanced photoaging Group (score from 71 to 100 and age between 60 and 85 years).

\subsection{Raman spectroscopy}

Raman spectra were obtained using a confocal Raman spectrometer (Model 3510; River Diagnostics BV) from the sunprotected (the middle third of the medial arm) and sun-exposed skin (the upper third of the dorsal forearm) of all subjects. The measurements were taken in the Laboratory of Biomedical Vibrational Spectroscopy, LEVB, at the Universidade do Vale do Paraíba, UniVap, in São José dos Campos, São Paulo, Brazil. The details of the instrument are described elsewhere [4]. Raman spectra were recorded in the spectral region from 600 to $1800 \mathrm{~cm}^{-1}$ using a $785 \mathrm{~nm}$ laser as an excitation source with power of around $20 \mathrm{~mW}$ at the sample. The subjects rested for 20 min in an acclimatised room before the Raman measurements. The studied sites were cleaned with $75 \%$ alcohol solution prior to each measurement. The temperature was maintained at $23 \pm 2{ }^{\circ} \mathrm{C}$ and the relative humidity at $51 \pm 5 \%$ during the measurements. Raman spectra were recorded in the spectral region from 600 to $1800 \mathrm{~cm}^{-1}$ using a $785 \mathrm{~nm}$ laser with power around $20 \mathrm{~mW}$. Measurements were initiated from a measurement template in the River-Icon ${ }^{\mathbb{R}}$ (River Diagnostics, Rotterdam, The Netherlands) software. The acquisition time used varied according to the depth analysed, as follows: from the skin surface to $12 \mu \mathrm{m}$ depth, $2 \mu \mathrm{m}$ 
increments and exposure time of $10 \mathrm{~s}$ each (one accumulation); from 12 to $80 \mu \mathrm{m}, 34 \mu \mathrm{m}$ increments and exposure time of $20 \mathrm{~s}$ each (one accumulation), from 80 to $120 \mu \mathrm{m}, 40 \mu \mathrm{m}$ increments and with three accumulations of $120 \mathrm{~s}$ each. In this work, spectra data analysis was considered only for the last two steps ( $80 \mu \mathrm{m}$ and $120 \mu \mathrm{m}$ ), corresponding to dermis measurements, as classified by cluster analysis (not shown).

\subsection{Raman data analysis}

Spectra analyses were carried out using Origin (OriginLab Corporation, Northampton, United States) and Labspec 5 (Horiba Jobin Yvon, Lille, France). The non-informative signals were first removed (spike removal) and the resulting spectra were filtered using a Savitsky-Golay filter (7 points, third order) in order to remove noisy features. The fluorescence-like spectral baseline was modelled using a linear baseline correction in standardised points (600, 794, 798, 994, 1144, 1494, 1716 and $1720 \mathrm{~cm}^{-1}$ ) and subsequently subtracted. Finally, Raman spectra were vectorial normalised.

\subsection{High frequency ultrasound}

A $20 \mathrm{MHz}$ unit (Dermascan C, Cortex Technology, Denmark) was used to obtain cross-sectional images of the skin, in triplicate by two trained investigators on the two studied regions. The echographic images were recorded and processed with image analysis software (Cortex Technology, Denmark) to calculate the number of medium and high echogenic pixels (MHEP), previously described elsewhere [13]. The mean value of the six measurements was used.

\subsection{Histology}

Histological and immunohistochemical evaluations of $4 \mathrm{~mm}$ punch biopsies from the same previously studied sites were followed by blind quantitative digital analysis. The picrosirius-red staining technique (total collagen) and immunohistochemical analysis of type I collagen (purified rabbit polyclonal anti-Collagen I antibody: Abcam Plc., code AB34710, United Kingdom) were used. The Verhoeff staining was also used for the photoexposed sections to highlight actinic elastosis. For digital quantitative analysis, pictures from the superficial dermis were captured in a standard way with a digital camera Moticam 3.0 MP (Motic, China) coupled to a light microscope ECLIPSE E200MV R (Nikon ${ }^{\circledR}$ Instruments, Japan). The digital images were processed using the software IMAGEJ (National Institutes of Health, Canada) to measure the area fraction occupied by collagen I (immunohistochemistry) and total collagen (picrosirius red-staining) as decribed by Brianezi et al. [14].

Table 1

Main clinical and demographic data from subjects.

\begin{tabular}{|c|c|c|c|}
\hline PHOTOAGING GROUPS & 1-LIGHT $(n=6)$ & 2- MODERATED $(\mathrm{n}=3)$ & 3-ADVANCED $(n=6)$ \\
\hline Age (years) ${ }^{a}$ & $35(28-48)$ & $56(54-60)$ & $68(62-82)$ \\
\hline \multicolumn{4}{|l|}{ Skin Phototype ${ }^{\mathrm{b}}$} \\
\hline II & $3(50)$ & $3(100)$ & $5(83)$ \\
\hline III & $3(50)$ & $0(0)$ & $1(17)$ \\
\hline Forearm photoaging score ${ }^{a}$ & $3(0-9)$ & $42(38-51)$ & $80(71-88)$ \\
\hline
\end{tabular}

a Mean (minimum- maximum).

b $\mathrm{n}(\%)$.

\subsection{Statistical analysis}

The spectra belonging to group 2 were not included in the first part of data analysis to highlight differences between the extreme groups. The remaining spectra were divided into 4 groups according to age and the photoaging scale: Group 1P: young (25 to 50 years), photoexposed region with light photoaging; Group 3P: elderly (60 to 85 years), photoexposed region presenting advanced photoaging; Group $1 \mathrm{~N}$ : young (25 to 50 years), nonphotoexposed region; and Group 3N: elderly (60 to 85 years), nonphotoexposed region. The averaged spectrum of each group was obtained for analysis of the peaks intensities and conformation. PCA $[15,16]$ and Orthogonal Partial Least Squares Discriminant Analysis (OPLS-DA) [17,18] were taken in the spectral range from $796 \mathrm{~cm}^{-1}$ to $996 \mathrm{~cm}^{-1}$ (proline and hydroxyproline region) to evaluate whether this region was capable of discriminating between the groups. This region has already been explored in other studies on skin aging and collagen and water interactions $[9,10,19]$.

For the second part of data analysis, the Group 2 spectra were included. The intensity of individual peaks $(855,875,922,938$, $1246,1275 \mathrm{~cm}^{-1}$ ) as well as the following peak intensities ratio were calculated: $\mathrm{I}_{938} / \mathrm{I}_{922}, \mathrm{I}_{855} / \mathrm{I}_{1450}, \mathrm{I}_{875} / \mathrm{I}_{1450}, \mathrm{I}_{922} / \mathrm{I}_{1450}, \mathrm{I}_{938} / \mathrm{I}_{1450}$, $\mathrm{I}_{1246} / \mathrm{I}_{1450}, \mathrm{I}_{1275} / \mathrm{I}_{1450}$. The first ratio $\left(\mathrm{I}_{938} / \mathrm{I}_{922}\right)$ may represent the amount of collagen-bound water according to previous studies [9]. The other ratios represent the intensity of the peaks in the numerator when the spectra are normalised by the peak at $1450 \mathrm{~cm}^{-1}$, which was chosen because it is not modified by alterations in secondary protein structure and does not take part in strong intermolecular interactions [7]. The results were used to evaluate the correlation between Raman spectroscopy and clinical score (photoexposed region), age, high frequency ultrasound parameters and histology (both in the photoexposed and in the non-photoexposed regions), using the Spearman rank correlation coefficient [20].

\section{Results}

Fifteen female subjects aged 28-82 years were enrolled in the study. They were divided into three groups according to the right forearm photoaging score (Table 1 ). A total of 30 dermal spectra ( $80 \mu \mathrm{m}$ and $120 \mu \mathrm{m}$ depth) from each site (photoexposed and nonphotoexposed skin) were collected. The spectra not classified as dermis spectra by cluster analysis were excluded. In the photoexposed region, 15 spectra were considered for final analysis (5 from group 1P, young, light photoaging; 4 from group 2P, moderate photoaging; and 6 from group 3P, elderly, advanced photoaging). In the non-photoexposed region, 16 spectra were considered for final analyses (6 from group 1N, young; 2 from group $2 \mathrm{~N}$; and 8 from group $3 \mathrm{~N}$, elderly). 


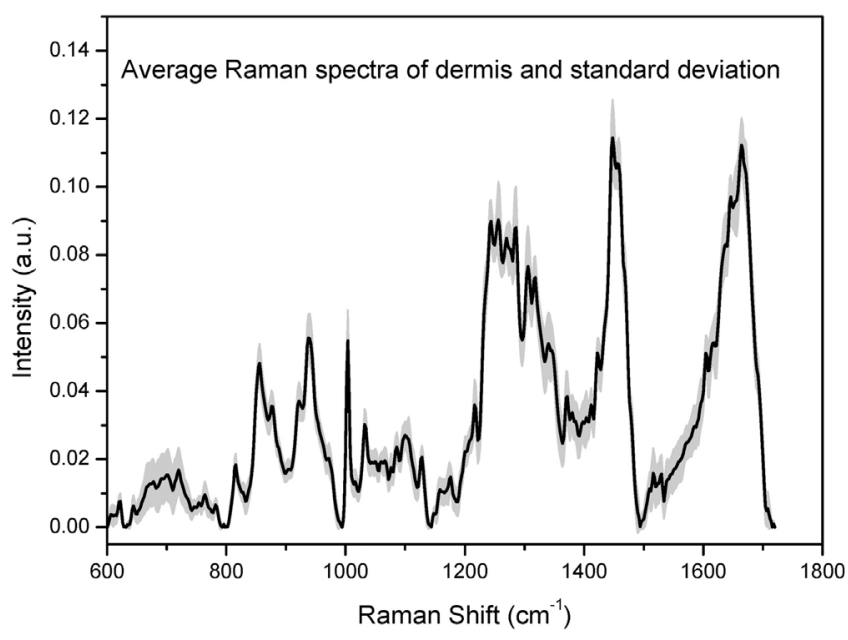

Fig. 1. Average and standard deviation of 31 dermis spectra.

Fig. 1 shows the average and standard deviation of the spectra from the dermis considered for analysis. It resembles collagen I Raman spectrum.

Fig. 2 shows the averaged spectra from each group (1P, 1N, 3P, $3 \mathrm{~N}$ ) and a closer view of the proline-hydroxyproline region. A visual analysis of the averaged spectra shows that the nonphotoexposed regions ( $1 \mathrm{~N}$ and $3 \mathrm{~N}$ ) present higher intensity values for the peak at $938 \mathrm{~cm}^{-1}$ than the photoexposed sites (1P and 3P). This peak intensity is also higher in the $3 \mathrm{~N}$ Group (elderly) compared to the $1 \mathrm{~N}$ Group (young). The other peaks $\left(855 \mathrm{~cm}^{-1}\right.$, $875 \mathrm{~cm}^{-1}$ and $922 \mathrm{~cm}^{-1}$ ) present higher intensity values for the $3 \mathrm{~N}$ Group than for the other groups, which present similar intensities.

PCA of the 25 spectra (first part of data analysis - Groups 1 and 3 ) was fitted with 4 principal components (PC) and coefficient of explained variance $\left(R^{2}\right)$ of $0.83\left(R^{2} P C 1=0.611, R^{2} P C 2=0.0961, R^{2}\right.$ $\left.\mathrm{PC} 3=0.0772, \mathrm{R}^{2} \mathrm{PC} 4=0.0457\right)$. PC1 vs. PC2 is plotted in Fig. 3. Data are colour-coded according to the corresponding group. PCA clusters are widely spread and not clearly differentiated, as expected for pure substances analysis [21]. This may be explained because all the measurements were taken from the dermis, which presents a complex molecular composition with high interindividual variability. Groups could not be discriminated either through plotting of PC1 vs. PC3, PC1 vs. PC4, PC2 vs. PC3, PC2 vs. PC4 and PC3 vs. PC4. To highlight possible differences between the groups, the data of pairs of groups were further submitted to PCA analysis, as follows:

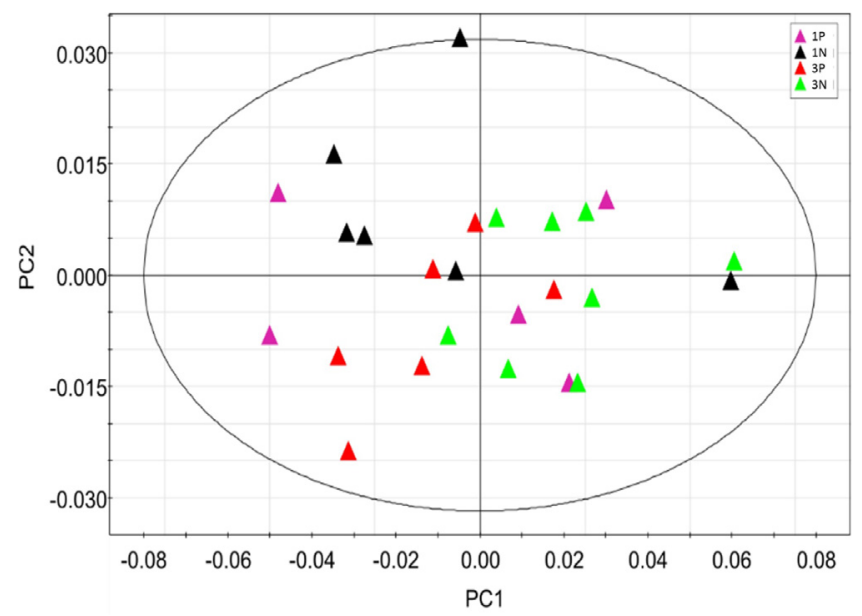

Fig. 3. PCA of the 25 Raman spectra - note PCA groups are widely spread and not clearly differentiated.

(i) intrinsic aging comparison (young inner arm vs. elderly inner arm): Group $1 \mathrm{~N}$ vs. Group 3N (Fig. 4a), with 2 PCs and $\mathrm{R}^{2}=0.78$.

(ii) advanced photoageing comparison (older inner arm vs. elderly forearm): Group 3N vs. Group 3P (Fig. 4b), with 3 PCs and $\mathrm{R}^{2}=0.78$

(iii) photoageing plus intrinsic aging comparison (young forearm vs. elderly forearm): Group 1P vs. Group 3P (Fig. 4c), with 2 PCs and $\mathrm{R}^{2}=0.69$.

(iv) light photoageing comparison (young inner arm vs. young forearm): Group 1N vs. Group 1P (Fig. 4d), with 2 PCs and $\mathrm{R}^{2}=0.80$.

As Fig. 4a shows, the young group can be discriminated from the older group through PC1 evaluation, and as Fig. 4b shows, the intrinsic aged skin can be discriminated from the extrinsic aged skin through PC1 analysis. However, the groups could not be discriminated in comparisons showed in Figs. 4c and d, i.e., 1P vs. $3 \mathrm{P}$ and $1 \mathrm{~N}$ vs. $1 \mathrm{P}$.

PCA is able to discriminate between groups when the intragroup variability is lower than the intergroup variability. Therefore, a supervised form of discriminant analysis (OPLS-DA) was also applied to the results to highlight the peaks that most contribute to the differences between the groups (Fig. 5). OPLS-DA of the 25 spectra resulted $R^{2} X=0.783$ and $R^{2} Y=0.88$, and the photoexposed and non-photoexposed sites are relatively well discriminated
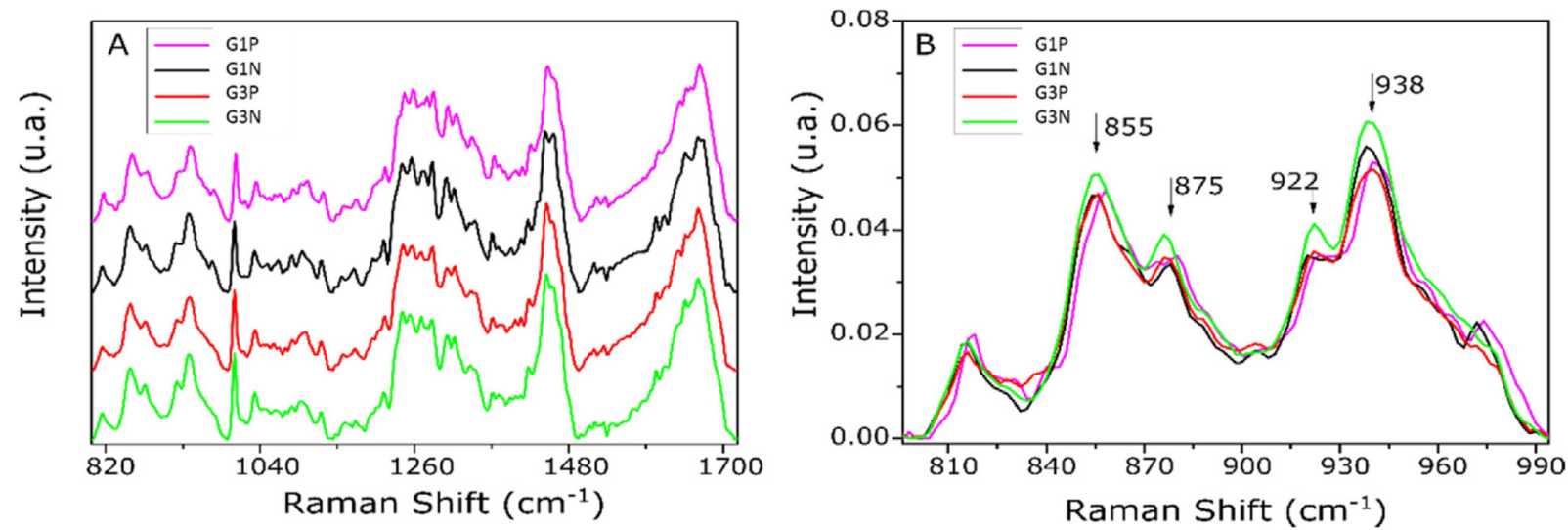

Fig. 2. A: the averaged Raman spectra of each group (1P, $1 \mathrm{~N}, 3 \mathrm{P}, 3 \mathrm{~N})$, B: with a closer view of the proline- hydroxyproline region. 

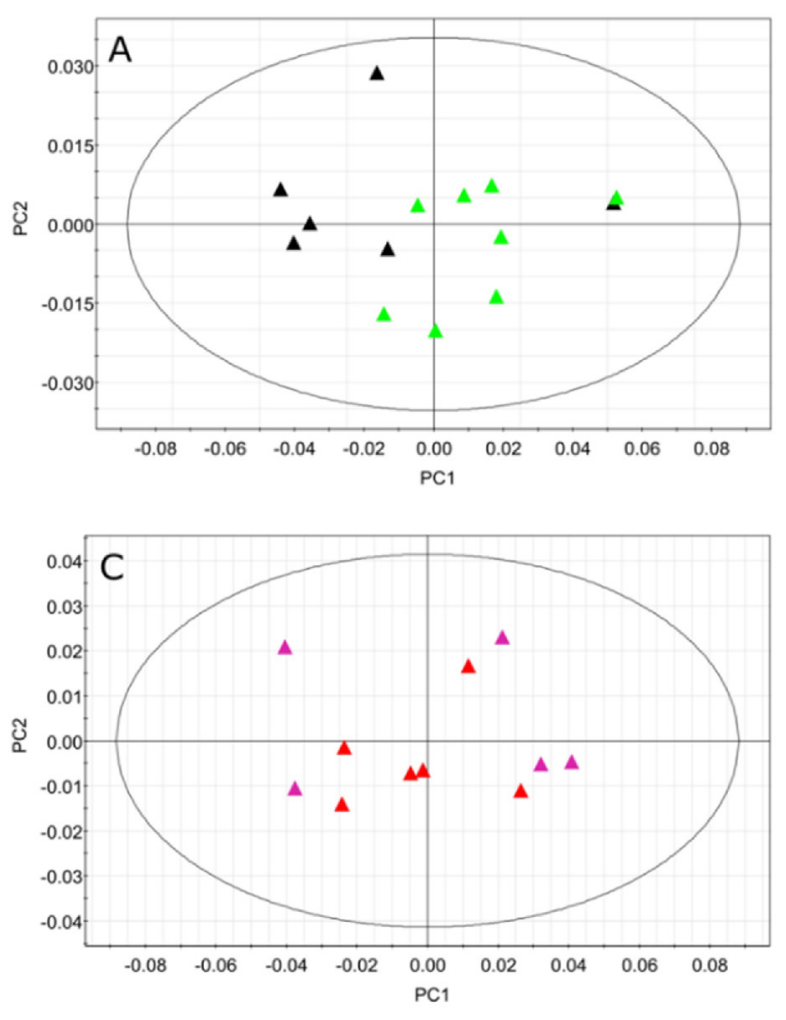
- 1P
- $1 \mathrm{~N}$
- 3P
$\triangle 3 N$

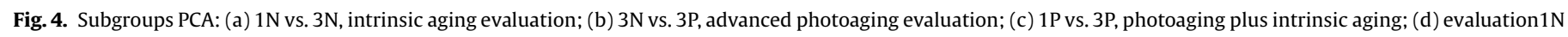
vs. 1P, light photoaging evaluation.

along the $\mathrm{X}$ axis using this method, as well as Groups $1 \mathrm{~N}$ and $3 \mathrm{~N}$ along the Y axis. The same is not true for Groups 1P and 3P, which are mixed (Fig. 5a). Fig. 5b shows the graph of variable importance in the projection (VIP) [22] and shows the important contribution of the $938 \mathrm{~cm}^{-1}$ band in the photoexposed vs. non-photoexposed discrimination. The VIP of to[1] axis (Y axis) - data not shown showed important contribution of the $938 \mathrm{~cm}^{-1}, 855 \mathrm{~cm}-1$, $922 \mathrm{~cm}-1$ e $875 \mathrm{~cm}-1$ bands (descending order) in the $1 \mathrm{~N}$ vs. $3 \mathrm{~N}$ discrimination.

In the second part of data analysis, Group 2 spectra were considered, in a total of 31 spectra. However, 7 volunteers presented more than 1 spectrum per site. In these cases, the averaged spectra were used. The photoexposed and the nonphotoexposed sites were represented by 12 spectra each. The intensities of selected peaks as well as some peak intensity ratios were calculated and the Spearman correlation coefficients between the Raman spectroscopy parameters and age, photoaging score, high frequency ultrasound and histology are shown in Tables 2 and 3 . The peaks that presented no statistically significant coefficients $\left(\mathrm{I}_{875}, \mathrm{I}_{922}, \mathrm{I}_{875} / \mathrm{I}_{1450}, \mathrm{I}_{922} / \mathrm{I}_{1450}\right)$ are not displayed in the table. The histology comparisons presented a lower number of cases because artefacts in some histological sections precluded digital image analysis.

In the non-photoexposed region, the intensity of the $855 \mathrm{~cm}^{-1}$ and $938 \mathrm{~cm}^{-1}$ bands presented moderate correlation with age and moderate to high inverse correlation with HFUS (MHEP UD/LD) and histology (collagen I IHC) (intrinsic aging assessment). The same did not occur in the photoexposed region. However, if the peak intensities are normalised by the peak intensity at $1450 \mathrm{~cm}^{-1}$, interesting correlations are prospected: the ratios $\mathrm{I}_{1246} / \mathrm{I}_{1450}$ and $\mathrm{I}_{1275} / \mathrm{I}_{1450}$ presented moderate to high correlation coefficients with age, photoaging score, HFUS echogenicity and histology (photoaging assessment).

\section{Discussion}

The results suggest that the wavenumber region between 798 and $994 \mathrm{~cm}^{-1}$ can be used to analyse the alterations that occur in dermal collagen through the intrinsic aging process and also the differences in dermal collagen between a non-photoexposed site (intrinsic aging alone) and advanced photoaged skin (extrinsic aging). However, the proline-hydroxyproline region seems not to be as useful in the assessment of the photoexposed region in different age groups, where intrinsic and extrinsic aging are superimposed. For this purpose, the $\mathrm{I}_{1275} / \mathrm{I}_{1450}$ ratio seems more proper.

In intrinsic aging, dermal fibroblasts show a less replicative capability and reduced type I procollagen synthesis. Increased levels of reactive oxygen species, decreased levels of antioxidants, higher expression of several matrix metalloproteinases (MMPs) and the accumulation of advanced glycosylation end products in dermal collagen result in catabolic effects [23-25]. Histopathologic studies on skin aging (abdominal skin) show that collagen bundles' thickness and their area fraction decrease with age, in both the papillary and reticular dermis, from 40 years of age onwards [23]. The elastic fibre network becomes gradually thinner and shorter and its total amount is reduced from 70 years onward [26], together with a decrease in the total sulfated glycosaminoglycans (GAGs) content [27].

Extrinsic aging, also called photoaging, occurs mainly due to the harmful effects of UVR, which induces the production of catabolic enzymes, such as MMPs, which cleaves the major interstitial collagens [23,24]. A gene expression profile study showed markedly increased expression of elastic fibre components in photoaged skin [24]. Histologic studies show reduced collagen staining replaced by elastotic material in the upper dermis in chronic sun-exposed skin [25]. Solar elastosis origin and 
$\triangle 1 P \triangle 1 N \quad \triangle 3 P \triangle 3 N$

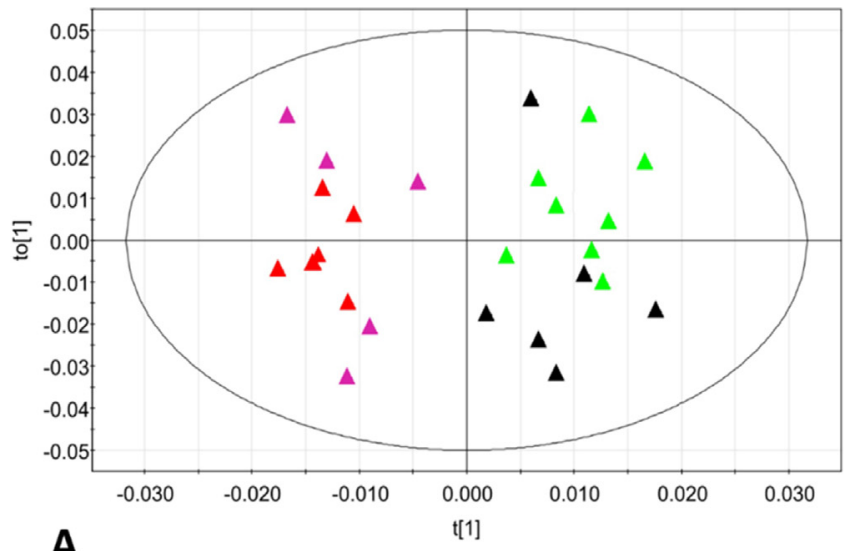

A

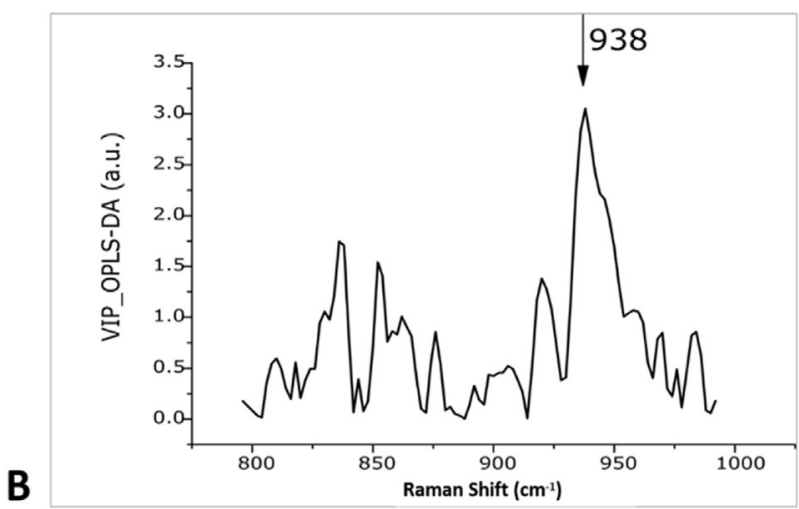

Fig. 5. OPLS-DA of the 25 spectra: the photoexposed and non-photoexposed sites are relatively well discriminated along $\mathrm{t}[1]$ axis, as well as are the groups $1 \mathrm{~N}$ and $3 \mathrm{~N}$ along to [1] axis. B: VIP ( $t$ [1] axis) shows the important contribution of the peak in $938 \mathrm{~cm}^{-1}$ in the photoexposed vs. non-photoexposed discrimination.

composition is controversial, but most evidence support a de novo synthesis of elastin, fibrillin and GAGs, although collagen and elastin degradation are also involved [28].

Because the main molecular constituent of the dermis is water (60\%) and type I collagen (85-90\% of dry weight) [29], the Raman spectra of the dermis closely resembles that of type I collagen, with important influence of collagen-water interactions [19]. Collagen is a macromolecule which consists of a set of three $\alpha$-helix polypeptide chains (tropocollagen) arranged in a triple-helical conformation. The polypeptide chain is characterised by a tri- aminoacid sequence of Glycine-X-Y type where $X$ usually corresponds to proline or $Y$ corresponds to hydroxyproline.

The Raman spectra of collagen and other peptide molecules show characteristic bands associated with the CONH group. In the low frequency region, amide I (stretching vibration of $\mathrm{C}=\mathrm{O}$ ) and amide III (associated with coupled $\mathrm{C}-\mathrm{N}$ stretching and $\mathrm{N}-\mathrm{H}$ bending vibrations of the peptide group) are useful for the identification of different protein backbone conformations and secondary structures [30]. In the amide III region, the vibrations at $1246 \mathrm{~cm}^{-1}$ and $1271 \mathrm{~cm}^{-1}$ are assigned to proline-rich and prolinepoor regions, respectively, and the intensity ratio $\mathrm{I}_{1271} / \mathrm{I}_{1246}$ reflects the proline residue proportion. This feature is associated with different spectral profiles in the $820-985 \mathrm{~cm}^{-1}$ range (prolinehydroxyproline region). The vibrations at 855 and $922 \mathrm{~cm}^{-1}$ are assigned to the proline ring and the peak at $875 \mathrm{~cm}^{-1}$ arises from the hydroxyproline ring. The $938 \mathrm{~cm}^{-1}$ band corresponds to the $\mathrm{C}-\mathrm{C}$ stretching vibration $(\nu \mathrm{C}-\mathrm{C})$ of the collagen backbone. $[10,30]$.

A previous FT-Raman spectroscopy study on skin aging analysed the high frequency region to assess water and protein content and also the amide I or amide III peak shift in the low frequency region according to intrinsic aging and photoaging [8]. Other studies analysed the proline-hydroxyproline region in ex vivo experiments $[10,19]$, evaluating intensity changes according to environmental relative humidity variations. They have demonstrated that the $938 \mathrm{~cm}^{-1}$ band (or the integrated intensity ratio between the bands in $938 \mathrm{~cm}^{-1}$ and $922 \mathrm{~cm}^{-1}-938 \mathrm{i} / 922 \mathrm{i}$ ) increases with increasing environmental relative humidity and decreases with increasing temperature (which leads to evacuation of free and bounded water). These findings suggest that this peak intensity provides a measure of the collagen-bound water content [10,19].

An in vivo experiment found that the ratio $938 \mathrm{i} / 922 \mathrm{i}$ was higher in elderly superficial dermis than in the young superficial dermis (ventral forearm) [11]. Similarly, our study showed higher intensity values for the $938 \mathrm{~cm}^{-1}$ band in the elderly group than in the young group in the non-photoexposed region. The results suggest that intrinsically aged skin presents better collagen hydration (bounded water) than young skin and is in accordance with other researches, which demonstrated that the water content is increased in the superficial dermis in intrinsic aging using an unilateral NMR scanner [31].

Although the correlation found between the $938 \mathrm{~cm}^{-1}$ peak intensity and age was not statistically significant (rho: 0.519 , $\mathrm{p}=0.08$ ), our results suggest an upward trend of this peak with intrinsic aging as rho value is positive. PCA of the groups $1 \mathrm{~N}$ and $3 \mathrm{~N}$ spectra corroborates with this finding because it shows that the proline-hydroxyproline region analysis discriminate them along

Table 2

Spearman rank correlation coefficients between Raman spectroscopy and age, HFUS and histology for the non-photoexposed region.

\begin{tabular}{|c|c|c|c|c|c|c|c|c|c|c|c|c|c|c|c|c|c|c|}
\hline \multirow{3}{*}{\multicolumn{2}{|c|}{$\begin{array}{l}\text { In vivo Raman } \\
\text { Spectroscopy vs. age, vs. } \\
\text { HFUS and vs. Histology }\end{array}$}} & \multicolumn{17}{|c|}{ Non-photoexposed } \\
\hline & & \multirow[t]{2}{*}{$\mathrm{N}$} & \multicolumn{2}{|l|}{$\mathrm{I}_{855}$} & \multicolumn{2}{|l|}{$\mathrm{I}_{938}$} & \multicolumn{2}{|l|}{$\mathrm{I}_{1246}$} & \multicolumn{2}{|l|}{$\mathrm{I}_{1275}$} & \multirow{2}{*}{$\frac{\mathrm{I}_{855} / \mathrm{I}_{1450}}{\text { rho }}$} & \multirow[b]{2}{*}{$p$} & \multicolumn{2}{|c|}{$\mathrm{I}_{938} / \mathrm{I}_{1450}$} & \multicolumn{2}{|c|}{$\mathrm{I}_{1246} / \mathrm{I}_{1450}$} & \multicolumn{2}{|c|}{$\mathrm{I}_{1275} / \mathrm{I}_{1450}$} \\
\hline & & & rho & $p$ & rho & $p$ & rho & $p$ & rho & $p$ & & & rho & $p$ & rho & $p$ & rho & $p$ \\
\hline Age & & 12 & 0.579 & 0.049 & 0.519 & 0.084 & -0.039 & 0.905 & -0.439 & 0.154 & 0.351 & 0.263 & 0.368 & 0.239 & -0.403 & 0.194 & -0.361 & 0.248 \\
\hline \multirow[t]{2}{*}{ MHEP/TP } & UD & 12 & -0.490 & 0.106 & -0.490 & 0.106 & -0.042 & 0.897 & 0.301 & 0.342 & 0.007 & 0.983 & -0.133 & 0.681 & 0.532 & 0.075 & 0.525 & 0.080 \\
\hline & $\mathrm{UD} / \mathrm{LD}$ & 12 & -0.678 & 0.015 & -0.710 & .0010 & -0.141 & 0.663 & 0.081 & 0.803 & -0.327 & 0.300 & -0.461 & 0.132 & 0.411 & 0.184 & 0.193 & 0.547 \\
\hline \multicolumn{2}{|c|}{ IHC COL I Area fraction (\%) } & 9 & $-\mathbf{0 . 7 0 0}$ & 0.036 & $-\mathbf{0 . 8 3 3}$ & 0.005 & 0.067 & 0.865 & 0.550 & 0.125 & -0.517 & 0.154 & $-\mathbf{0 . 7 0 0}$ & 0.036 & 0.583 & 0.099 & 0.867 & 0.003 \\
\hline \multicolumn{2}{|c|}{$\begin{array}{l}\text { Picrosirius Area fraction } \\
(\%)\end{array}$} & 11 & 0.009 & 0.979 & -0.018 & 0.958 & 0.418 & 0.201 & 0.373 & 0.259 & -0.255 & 0.450 & -0.373 & 0.259 & 0.064 & 0.853 & -0.027 & 0.937 \\
\hline
\end{tabular}

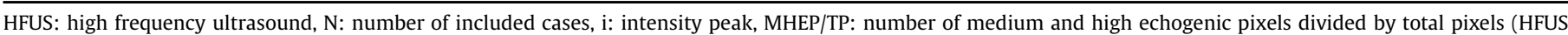
parameter), UD: upper dermis, LD: lower dermis, IHC COL I: collagen I immunohistochemistry.

Bold values signify $\mathrm{p}<0.05$. 
Table 3

Spearman rank correlation coefficients between Raman spectroscopy and age, clinical score, HFUS and histology for the photoexposed region.

\begin{tabular}{|c|c|c|c|c|c|c|c|c|c|c|c|c|c|c|c|c|c|}
\hline \multirow{3}{*}{$\begin{array}{l}\text { In vivo Raman } \\
\text { Spectroscopy vs. age, vs. } \\
\text { clinical score, vs. HFUS and } \\
\text { vs. Histology }\end{array}$} & \multicolumn{17}{|c|}{ Photoexposed } \\
\hline & \multirow[t]{2}{*}{$\mathrm{N}$} & \multicolumn{2}{|l|}{$\mathrm{I}_{855}$} & \multicolumn{2}{|l|}{$\mathrm{I}_{938}$} & \multicolumn{2}{|l|}{$\mathrm{I}_{1246}$} & \multicolumn{2}{|l|}{$\mathrm{I}_{1275}$} & \multirow{2}{*}{$\frac{\mathrm{I}_{855} / \mathrm{I}_{1450}}{\text { rho }}$} & \multirow[b]{2}{*}{$p$} & \multicolumn{2}{|c|}{$\mathrm{I}_{938} / \mathrm{I}_{1450}$} & \multicolumn{2}{|c|}{$\mathrm{I}_{1246} / \mathrm{I}_{1450}$} & \multicolumn{2}{|c|}{$\mathrm{I}_{1275} / \mathrm{I}_{1450}$} \\
\hline & & rho & $p$ & rho & $p$ & Rho & $p$ & rho & $p$ & & & rho & $p$ & rho & $p$ & rho & $p$ \\
\hline Age & 12 & -0.133 & 0.680 & -0.235 & 0.462 & -0.084 & 0.795 & -0.533 & 0.074 & -0.407 & 0.189 & -0.393 & 0.206 & -0.375 & 0.229 & -0.730 & 0.007 \\
\hline Photoaging clinical score & 12 & -0.180 & 0.575 & -0.290 & 0.361 & -0.141 & 0.661 & -0.505 & 0.094 & -0.406 & 0.190 & -0.385 & 0.216 & -0.339 & 0.281 & -0.594 & 0.042 \\
\hline MHEP/TP & 12 & 0.329 & 0.297 & 0.385 & 0.217 & 0.357 & 0.255 & 0.636 & 0.026 & 0.678 & 0.015 & .580 & 0.048 & 0.790 & 0.002 & 0.860 & $\mathbf{0 . 0 0 0}$ \\
\hline $\mathrm{UD} / \mathrm{LD}$ & 12 & 0.179 & 0.578 & 0.319 & 0.312 & 0.375 & 0.229 & 0.765 & 0.004 & 0.291 & 0.358 & 0.347 & 0.269 & 0.509 & 0.091 & 0.754 & 0.005 \\
\hline IHC COL I Area fraction (\%) & 12 & -0.217 & 0.499 & -0.112 & 0.729 & 0.161 & 0.618 & 0.559 & 0.059 & 0.035 & 0.914 & -0.035 & 0.914 & 0.483 & 0.112 & 0.643 & 0.024 \\
\hline Picrosirius Area fraction (\%) & 11 & 0.027 & 0.937 & 0.064 & 0.853 & 0.473 & 0.142 & 0.664 & 0.026 & 0.118 & 0.729 & 0.100 & 0.770 & 0.636 & 0.035 & 0.773 & 0.005 \\
\hline Verhoef Area fraction (\%) & 11 & 0 & ns & -0.100 & 0.770 & -0.564 & 0.071 & -0.818 & 0.002 & -0.127 & 0.709 & 0.100 & 0.770 & -0.709 & 0.015 & -0.727 & 0.011 \\
\hline
\end{tabular}

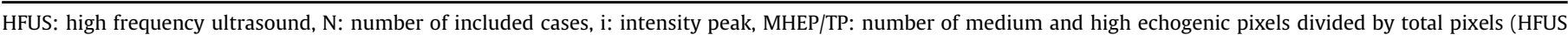
parameter), UD: upper dermis, LD: lower dermis, IHC COL I: collagen I immunohistochemistry.

Bold values signify $\mathrm{p}<0.05$.

the PC1. The OPLS-DA VIP graph of to[1] axis (data not shown) shows important contribution of the $938 \mathrm{~cm}^{-1}$ and $855 \mathrm{~cm}^{-1}$ peaks for the same groups differentiation along to[1]. This last peak intensity presented significant correlation with age (rho: 0,579 , $\mathrm{p}=0,049$ ) in the non-photoexposed region.

Our results also suggest that collagen in intrinsically aged skin $(3 \mathrm{~N})$ is better hydrated than in photoaged skin (3P), even though previous studies have shown the amount of bulk water (nonmacromolecule bounded water) to be increased in the photoexposed sites $[7,8]$. However, the spectra regions that evaluates free water content $\left(180 \mathrm{~cm}^{-1}\right)$ and water-protein proportion $\left(2600-4000 \mathrm{~cm}^{-1}\right)$ were not studied in our experiment. PCA carried out with the subgroups $3 \mathrm{~N}$ vs. $3 \mathrm{P}$ shows that they can be discriminated through PC1 evaluation.

These collagen hydration trends resemble the proportion of hydroxyproline in the dermis during the intrinsic aging and photoaging processes. Previous researches demonstrated that while intrinsic aging is characterised by an increase in the hydroxyproline content when compared to young skin, chronically sun-damaged skin actually shows a decrease in hydroxyproline content [32]. The collagen fibre structure is stabilised by means of indirect intra- and inter-chain hydrogen bonds involving water molecules, the carbonyl groups of every amino acid and/or the hydroxyl groups of hydroxyproline residues $[10,19]$. Thus, the proportion of hydroxyproline residues plays an important role in collagen hydration.

The fact that PCA was not able to explain the differences between the groups $1 \mathrm{~N}$ and $1 \mathrm{P}$ (Fig. 5c) was already expected, because this group presented low values of forearm photoaging score, and previous studies have already showed they present similar protein and water structure [8]. PCA was not able to discriminate groups $1 \mathrm{P}$ and $3 \mathrm{P}$ either (Fig. 5d). This comparison reflects both intrinsic (age-related) and extrinsic aging. However, hydroxyproline residues present opposite behaviour throughout the two types of skin aging: rising and decreasing values; and this may have influenced the final results. Another hypothesis to explain this is the huge structure alteration in collagen molecule in photoaging and its replace by elastotic material, which may influence the amide I and III regions and their contribution to the final spectra when it is submitted to vectorial normalisation. Also, the decrease in the total collagen content is in agreement with lower intensity peaks in the proline-hydroxyproline region for the photoexposed sites in both groups.

Gniadecka et al. [8] showed the chronically photoexposed skin to present secondary protein structure alterations resulting in a downshift of amide I and amide III peaks. Data normalisation for the $1450 \mathrm{~cm}^{-1}$ band intensity was preferred in their study because it is a $\mathrm{C}-\mathrm{H}$ band, which protrudes outside the protein chain and is not influenced by alterations in secondary protein structure. In fact, when our data are normalised for this band (dividing the intensity of the peak of interest by the intensity of the peak in $1450 \mathrm{~cm}^{-1}$ ), direct correlations are detected between $\mathrm{I}_{1275} / \mathrm{I}_{1450}$ ratio and age and also the photoaging score.

In the non-photoexposed regions, the intensity of the bands in $855 \mathrm{~cm}^{-1}$ and $938 \mathrm{~cm}^{-1}$ presented low to moderate inverse correlation with the amount of collagen I in histology and also echogenicity in HFUS. These results are in accordance with other studies that demonstrated that $938 \mathrm{~cm}^{-1}$ band intensity increases with age in the superficial dermis [11] and correlates directly with collagen-bound water [19]. The higher amount of bound water may justify the thinner collagen fibres with a reduced area fraction seen in intrinsic aging histologic studies [23]. The picrosirius red staining disclosed no significant correlation with Raman spectroscopy probably because it marks both types of collagen (type I and type III) and intrinsic aging is mainly characterised by collagen I reduction $[8,24]$.

In the photoexposed region, the same band intensities $855 \mathrm{~cm}^{-1}$ and $938 \mathrm{~cm}^{-1}$ presented no correlation with age, photoaging score, HFUS and histology. However, when spectra are normalised for the $1450 \mathrm{~cm}^{-1}$ intensity peak $\left(\mathrm{I}_{855} / \mathrm{I}_{1450}, \mathrm{I}_{938} /\right.$ $\mathrm{I}_{1450}$ ) direct correlations are found between them and echogenicity - the opposite that occurs in the non-photoexposed region, in agreement with the opposite hydroxyproline behaviour through intrinsic aging and photoaging. This results also agree with a previous Raman spectroscopy study of porcine heart valves before and after treatment with collagenase, which showed significant reduction of the following peaks intensities $938 \mathrm{~cm}^{-1}, 1244 \mathrm{~cm}^{-1}$, $1272 \mathrm{~cm}^{-1}$ and $1664 \mathrm{~cm}^{-1}$ [33]. Interestingly, the $\mathrm{I}_{1246} / \mathrm{I}_{1450}$ and $\mathrm{I}_{1275} / \mathrm{I}_{1450}$ intensity ratios presented moderate to strong correlation coefficients with HFUS and histology parameters in our study in the photoexposed region.

Previous studies reveled minimal contribution of elastin to the Raman spectra of normal skin [8], but a greater contribution should be expected in the photoaged dermis due to elastotic material deposition. The vibrations at $1246 \mathrm{~cm}^{-1}$ and $1274 \mathrm{~cm}^{-1}$ also contribute to the Raman spectra of pure elastin [34,35]. The Verhöff staining area fraction presented the higher correlation coefficient between the $\mathrm{I}_{1246} / \mathrm{I}_{1450}$ and histology. This may suggest a higher contribution of elastin to the Raman spectra of the photoaged dermis. However, the correlation is inverse (negative signal) and the final spectra of the dermis from the photoexposed region resembles collagen spectra (and not elastin spectra). 
Although GAGs and proteoglycans also account for a minimal component of the normal dermis, the amount of total sulfatated GAGs also raises inside the elastotic material during the photoaging process [36]. So, a higher contribution to the Raman spectra may also be expected in this case. However, studies to elucidate the influence of GAGs in the Raman spectra of the skin are missing.

Our study suggests the intensity ratio $\mathrm{I}_{1275} / \mathrm{I}_{1450}$ is a promising parameter to be explored in further photoaging studies, while the proline-hydroxyproline region $\left(855 \mathrm{~cm}^{-1}\right.$ and $938 \mathrm{~cm}^{-1}$ band intensities) are suitable for intrinsic aging assessment. This is the first skin aging study to show a correlation between Raman peaks and the amount of collagen assessed by HFUS and histology.

Future Raman microspectroscopy studies on photoaged skin histologic sections may give further support to our findings. However, the high correlations found between in vivo Raman spectroscopy and histology in this study suggest that it is promising technique to reduce the need for invasive biopsies in future studies. Similar protocols may evaluate the ability of this method in collagen assessment in other entities such as scleroderma and keloid scars.

\section{Funding}

FUNADERSP, an organisation of the São Paulo regional office of the Brazilian Society of Dermatology with grant number 001/2013.

\section{Conflict of interest}

The authors have no conflict of interest to declare.

\section{Acknowledgements}

This work was supported by FUNADERSP, an organisation of the Sao Paulo regional office of the Brazilian Society of Dermatology SBD-RESP (project no. 001/2013). AAM thanks to CNPq (307809/ 2013-7).

We would like to thank the following individuals for giving support in the Raman spectroscopy measurements in the Laboratory of Biomedical Vibrational Spectroscopy at the Universidade do Vale do Paraíba: Débora Braga Isensee, Thais Cristina da Silva and Mariane Musskopf.

\section{References}

[1] Z. Movasaghi, S. Rehman, I.U. Rehman, Raman spectroscopy of biological tissues, Appl. Spectrosc. Rev. 42 (2007) 493-541.

[2] S. Olsztyńska-Janus, M. Gąsior-Głogowska, K. Szymborska-Małek, M. Komorowska, W. Witkiewicz, C. Pezowicz, S. Szotek, M. Kobielarz, Spectroscopic techniques in the study of human tissues and their components. Part II: Raman spectroscopy, Acta Bioeng. Biomech. 14 (2012) 121-133.

[3] M.G. Shim, B.C. Wilson, Development of an in vivo Raman spectroscopic system for diagnostic applications, J. Raman Spectrosc. 28 (1997) 131-142.

[4] P.J. Caspers, G.W. Lucassen, R. Wolthuis, H.A. Bruining, G.J. Puppels, In vitro and in vivo Raman spectroscopy of human skin, Biospectroscopy 4 (1998) S31-S39.

[5] P. Caspers, G. Lucassen, H. Bruining, G. Puppels, Automated depth-scanning confocal Raman microspectrometer for rapid in vivo determination of water concentration profiles in human skin, J. Raman Spectrosc. 31 (2000) 813-818.

[6] C. Choe, J. Lademann, M.E. Darvin, Confocal Raman microscopy for investigating the penetration of various oils into the human skin in vivo, J. Dermatol. Sci. 79 (2015) 171-178.

[7] N. Nakagawa, M. Matsumoto, S. Sakai, In vivo measurement of the water content in the dermis by confocal Raman spectroscopy, Skin Res. Technol. 16 (2010) 137-141.

[8] M. Gniadecka, O.F. Nielsen, S. Wessel, M. Heidenheim, D.H. Christensen, H.C. Wulf, Water and protein structure in photoaged and chronically aged skin, J. Invest. Dermatol. 111 (1998) 1129-1133.

[9] F.J. González, C. Castillo-Martínez, M. Martínez-Escanamé, M.G. Ramírez-Elías, F.I. Gaitan-Gaona, C. Oros-Ovalle, B. Moncada, Non-invasive estimation of chronological and photo-induced skin damage using Raman spectroscopy and principal component analysis, Skin Res. Technol. 18 (2012) 442-446.
[10] T. Nguyen, J. Happillon, J.F. Brassart-Passco, M. Angiboust, O. Piot, Raman comparison of skin dermis of different ages: focus on spectral markers of collagen hydration, J. Raman Spectrosc. 44 (2013) 1230-1237.

[11] S.L. Téllez, L. dos Santos, P. Fávero, A.A. Martin, RM1 semi empirical and DFT: B3LYP/3-21G theoretical insights on the confocal Raman experimental observations in qualitative water content of the skin dermis of healthy young, healthy elderly and diabetic elderly women's, Spectrochim. Acta A Mol. Spectrosc. 149 (2015) 1009-1019.

[12] C.O. Guimarães, E. Bagatin, L. Guadanhim, F. Sternberg, F.R. Picosse, G. Nunes, C. Milanez, H.A. Miot, Development and validation of a clinical scale for the evaluation of forearm skin photoaging, J. Cutaneous Med. Surg. 19 (2015) 380387.

[13] L. de, V.N. Caetano, J.L. Soares, E. Bagatin, H.A. Miot, Reliable assessment of forearm photoageing by high-frequency ultrasound: a cross-sectional study, Int. J. Cosmet. Sci. 38 (2016) 170-177.

[14] G. Brianezi, F. Grandi, E. Bagatin, M.M. Enokihara, H.A. Miot, Dermal type I collagen assessment by digital image analysis, An. Bras. Dermatol. 90 (2015) 723-727.

[15] S. Wold, K. Esbensen, P. Geladi, Principal component analysis, Chemom. Intell. Lab. Syst. 2 (1987) $37-52$.

[16] I.T. Jolliffe, Principal Component Analysis, second ed., Springer-Verlag, New York, 2002.

[17] J. Trygg, S. Wold, Orthogonal projections to latent structures (O-PLS), J. Chemom. 16 (2002) 119-128.

[18] M. Bylesjö, M. Rantalainen, O. Cloarec, J.K. Nicholson, E. Holmes, J. Trygg, OPLS discriminant analysis: combining the strengths of PLS-DA and SIMCA classification, J. Chemom. 20 (2006) 341-351.

[19] Q. Zhang, K.L. Andrew Chan, G. Zhang, T. Gillece, L. Senak, D.J. Moore, R. Mendelsohn, C.R. Flach, Raman micro spectroscopic and dynamic vapour sorption characterization of hydration in collagen and dermal tissue, Biopolymers 95 (2011) 607-615.

[20] R. Artusi, P. Verderio, E. Marubini, Bravais-Pearson and Spearman correlation coefficients: meaning: test of hypothesis and confidence interval, Int. J. Biol. Markers 17 (2002) 148-151.

[21] F. Bonnier, H.J. Byrne, Understanding the molecular information contained in principal component analysis of vibrational spectra of biological systems, Analyst 137 (2012) 322-332.

[22] N.L. Afanador, T.N. Tran, L.M.C. Buydens, Use of the bootstrap and permutation methods for a more robust variable importance in the projection metric for partial least squares regression, Anal. Chim. Acta 768 (2013) 49-56.

[23] V. Marcos-Garcés, P. Molina Aguilar, C. Bea Serrano, V. García Bustos, J. Benavent Seguí, A. Ferrández Izquierdo, A. Ruiz-Saurí, Age-related dermal collagen changes during development, maturation and ageing - a morphometric and comparative study, J. Anat. 225 (2014) 98108.

[24] J.A. McGrath, M.K. Robinson, R.L. Binder, Skin differences based on age and chronicity of ultraviolet exposure: results from a gene expression profiling study, Br. J. Dermatol. 166 (Suppl. 2) (2012) 9-15.

[25] K. Kawabata, M. Kobayashi, A. Kusaka-Kikushima, E. Akasaka, T. Mabuchi, T. Fukui, Y. Sugiyama, S. Takekoshi, M. Miyasaka, A. Ozawa, S. Sakai, A new objective histological scale for studying human photoaged skin, Skin Res. Technol. 20 (2014) 155-163.

[26] M. El-Domyati, S. Attia, F. Saleh, D. Brown, D.E. Birk, F. Gasparro, et al., Intrinsic aging vs. photoaging: a comparative histopathological, immunohistochemical, and ultrastructural study of skin, Exp. Dermatol. 11 (2002) 398-405.

[27] J.H. Oh, Y.K. Kim, J.Y. Jung, J.E. Shin, K.H. Kim, K.H. Cho, et al., Intrinsic agingand photoaging-dependent level changes of glycosaminoglycans and their correlation with water content in human skin, J. Dermatol. Sci. 62 (2011) 192201.

[28] K. Sellheyer, Pathogenesis of solar elastosis: synthesis or degradation? J. Cutaneous Pathol. 30 (2003) 123-127.

[29] J.A. McGrath, J. Uitto, Anatomy and organization of human skin, in: T. Burns, S. Breathnach, N. Cox, C. Griffiths (Eds.), Rook's Textbook of Dermatology, eighth ed., Wiley-Blackwell, Oxford, 2010 pp. 3.1-3.53.

[30] A. Rygula, K. Majzner, K.M. Marzec, A. Kaczor, M. Pilarczyk, M. Baranska, Raman spectroscopy of proteins: a review, J. Raman Spectrosc. 44 (2013) 1061-1076.

[31] E. Bergman, Y. Sarda, N. Ritz, E. Sabo, G. Navon, R. Bergman, U. Nevo, In vivo assessment of aged human skin with a unilateral NMR scanner, NMR Biomed. 28 (2015) 656-666.

[32] J.G. Smith Jr., E.A. Davidson, W.M. Sams Jr., R.D. Clark, Alterations in human dermal connective tissue with age and chronic sun damage, J. Invest. Dermatol. 39 (1962) 347-350.

[33] M. Votteler, D.A. Carvajal Berrio, M. Pudlas, H. Walles, U.A. Stock, K. SchenkeLayland, Raman spectroscopy for the non-contact and non-destructive monitoring of collagen damage within tissues, J. Biophotonics 5 (2012) 47-56.

[34] L. Debelle, A.J. Alix, S.M. Wei, M.P. Jacob, J.P. Huvenne, M. Berjot, et al., The secondary structure and architecture of human elastin, Eur. J. Biochem. 258 (1998) 533-539.

[35] E. Green, R. Ellis, P. Winlove, The molecular structure and physical properties of elastin fibers as revealed by Raman microspectroscopy, Biopolymers 89 (2008) 931-940.

[36] E.F. Bernstein, C.B. Underhill, P.J. Hahn, D.B. Brown, J. Uitto, Chronic sun exposure alters both the content and distribution of dermal glycosaminoglycans, Br. J. Dermatol. 135 (1996) 255-262. 